\title{
Development and Approbation of Methodology Aimed to Define the Reasons for Turbine Unit Capacity Limitation Based on the Specified Mathematical Model of its Condenser \\ Shempelev A. G. \\ Vyatka State University \\ Kirov, Russian Federation
}

\begin{abstract}
The purpose of this work is to develop and test the methodology of elucidation of the reasons for turbine unit capacity limitations based on a mathematical model of its condenser. This purpose is achieved by using a mathematical model of the condenser as part of the developed methodology, taking into account the separate effects of contamination of the heat exchange surfaces, air suction into the vacuum system and the operating mode of the main ejector. Based on operational data sampling, the value of the limiting pressure in the condenser, excess of which leads to limitation of turbine unit capacity, was determined. It was established that the cause of power limitation is the abnormal operation of the main ejector due to inadmissible high temperature in the intermediate cooler of its first stage. For regimes that were not pressure-limited, using a mathematical model, the degree of tubes contamination, its influence on the condenser pressure and the power generated by the turbine unit, and the influence of actual air suctions on the condenser pressure were determined. The most important result of the study is to determine the possibility and feasibility of using the developed and tested methodology for solving similar problems for any type of turbine unit equipped with a condenser. The significance of the work lies in the fact that the proposed approach expands the possibilities of using mathematical models of this class in terms of solving such problems.
\end{abstract}

Keywords: steam turbine, capacity, condenser, main ejector, limitations, condenser pressure, mathematical model of a condenser.

DOI: https://doi.org/10.52254/1857-0070.2021.4-52.08

UDC: 62.173

Elaborarea și aprobarea metodologiei pentru determinarea motivelor limitărilor de putere ale turboinstalației în baza modelului matematic rafinat al condensatorului acesteia

Șempelev A.G.

Universitatea de Stat din Viatca

Kirov, Federația Rusă

Rezumat. Actualmente, modelele matematice ale condensatoarelor turbinelor cu aburi sunt utilizate pe larg pentru diagnosticarea stării instalațiilor ce funcționează în baza condensării, dar lipsesc metodologiile de aplicare pentru depistarea motivelor de putere electrică limitată, inclusiv, după presiunea în condensator. Scopul lucrării este elaborarea și aprobarea metodologiei de elucidare a motivelor limitărilor de putere a instalației turbinei în baza unui model matematic al condensatorului acestuia. Acest scop este atins prin utilizarea în componența metodologiei modelului matematic al condensatorului, luând în considerare efectele separate ale impurificării suprafețelor de schimb de căldură, aspirarea aerului în sistemul de vid și regimul de funcționare al ejectorului principal al jetului de abur. Pentru regimurile nelimitate de presiune, folosind modelul matematic s-a determinat gradul de impurificare a tuburilor, influența acestuia asupra presiunii în condensator și a puterii generate de instalația turbinei, precum și influența aspirațiilor reale de aer și asupra presiunii condensatorului. Calculele dependenţelor presiunii de consumul de aburi în condensator la diferite temperaturi ale apei de răcire la ieșirea din condensator au arătat că generarea puterii nominale în condiţiile examinate este posibilă la temperaturile apei mai jos de $25^{\circ} \mathrm{C}$. Cel mai important rezultat al studiului este determinarea posibilităţii și raţionalităţii utilizării metodologiei elaborate şi aprobate pentru soluționarea unor probleme similare pentru orice tip de instalație turbină echipată cu condensator. Semnificația lucrării constă în faptul, că abordarea propusă extinde posibilitățile de utilizare a modelelor matematice ale acestei clase în ceea ce privește soluționarea unor astfel de probleme.

Cuvinte-cheie: turbină cu abur, capacitate, condensator, ejector principal, limitări, presiunea condensatorului, modelul matematic al unui condensator.

Разработка и апробация методики выяснения причин ограничений мощности турбоустановки на основе уточненной математической модели ее конденсатора

Шемпелев А. Г.

Вятский государственный университет

Киров, Российская Федерация

Аннотация. В настоящее время математические модели конденсаторов паровых турбин широко используются для диагностики состояния самих конденсационных установок, но отсутствуют методики 
их применения для определения причин ограничений электрической мощности, в том числе по давлению в конденсаторе. Целью данной работы является разработка и апробация методики выяснения причин ограничений мощности турбоустановки на основе математической модели ее конденсатора. Указанная цель достигается путем использования в составе разработанной методики математической модели конденсатора, учитывающей раздельное влияния загрязнений поверхностей теплообмена, присосов воздуха в вакуумную систему и режима работы основного пароструйного эжектора. В соответствии с этой методикой, на основе эксплуатационных данных определена величина давления, превышение которого приводит к ограничению мощности турбоустановки. По величине предельного давления определена величина допустимого расхода пара в конденсатор. Установлено, что причиной появления ограничений мощности является нештатная работа основного эжектора вследствие недопустимо высокой температуры в промежуточном охладителе его первой ступени. Для режимов, не ограниченных по давлению, с помощью математической модели определена степень загрязнения трубок, ее влияние на давление в конденсаторе и на вырабатываемую турбоустановкой мощность, а также влияние фактических присосов воздуха на давление в конденсаторе. Расчеты зависимостей давления от расхода в пара в конденсатор при различных температурах охлаждающей воды на входе в конденсатор показали, что выработка номинальной мощности в рассматриваемых условиях возможна при температурах воды ниже $25^{\circ} \mathrm{C}$. Наиболее важным результатом исследования является определение возможности и целесообразности применения разработанной и апробированной методики для решения аналогичных задач для любых типов турбоустановок, оснащенных конденсатором. Значимость работы заключается в том, что предлагаемый подход расширяет возможности применения математических моделей данного класса в части решения такого рода задач.

Ключевые слова: паровая турбина, мощность, конденсатор, главный эжектор, ограничения, давление в конденсаторе, математическая модель конденсатора.

\section{ВВЕДЕНИЕ}

Сокращение потребления пара производственного отбора или полное его отсутствие вызывает определенные проблемы при эксплуатации турбин с производственным отбором пара (турбины типа ПТ). Особенно остро такие проблемы возникают на ТЭЦ, принадлежащих крупным металлургическим и химическим предприятиям, в неотопительный период при необходимости круглогодичной выработки максимального количества электрической энергии, то есть когда возникает необходимость эксплуатации турбоустановок типа ПТ на конденсационном режиме. Основной проблемой при этом является ограничение мощности по давлению в конденсаторе [1-4].

В инструкциях по эксплуатации большинства турбоустановок обычно указана величина предельно допустимого давления в конденсаторе в эксплуатационных условиях, и это давление обычно составляет 12 кПа. Для турбоагрегатов среднего давления и с ухудшенным вакуумом возможны и другие ограничивающие значения величин давлений отработавшего пара. Выбор этой величины связан с обеспечением надежной работы турбинных ступеней, термомеханическим состоянием части низкого давления и обеспечением надежной работы основных эжекторов [5].
Ограничения мощности могут возникнуть или вследствие несоответствия параметров циркуляционной системы (количества и температуры охлаждающей воды) конденсационным нагрузкам турбоагрегатов [6-8], или из-за ухудшения характеристик конденсатора и основных эжекторов в процессе эксплуатации [9-14].

Наличие ограничений мощности по давлению в конденсаторе может быть установлено в рамках систем мониторинга состояния оборудования низкопотенциального комплекса и его диагностики. Этой проблеме посвящен ряд работ, в которых рассматриваются причины отклонения указанных выше факторов и их влияние на располагаемую мощность турбоустановки $[6,8,9]$. К настоящему времени разработаны различные системы мониторинга, которые могут быть встроены в систему автоматизированного управления технологическим процессом (АСУ ТП) [6, 15-19]. Использование таких систем предполагает, в том числе, решение постоперативных задач, включающих диагностирование вспомогательного оборудования и техникоэкономические задачи $[1,20,21]$.

Одно из центральных мест в системах диагностики оборудования занимают используемые в них математические модели, в частности, математические модели конденсаторов, предусматривающие вполне определенные 
цифровые алгоритмы определения выявляемого дефекта [22, 23]. Цифровые алгоритмы не связаны с типом задач, конкретным оборудованием, его особенностями, режимами работы. От типа алгоритма зависит степень определенности выявляемого дефекта [24].

Проведенный анализ опубликованных результатов зарубежных исследований [6, 15-23] показал, что в них исследуются возможности диагностики определенных дефектов и несоответствий в работе турбоустановок, но, практически, отсутствуют данные по использованию математических моделей конденсаторов для решения задач, связанных с определением допустимых давлений в конденсаторах с учетом величин присосов воздуха и характера взаимодействия конденсатора и основного эжектора в широком диапазоне его тепловых нагрузок.

Поэтому определение конденсационных режимов работы турбоустановок, обеспечивающих минимальные ограничения мощности, на основе математической модели конденсатора, учитывающей указанные факторы, является новой и весьма актуальной задачей, которая и решается в данном исследовании.

Целью работы является разработка и апробация отсутствовавшей ранее методики выяснения причин ограничений мощности турбоустановки на основе математической модели ее конденсатора с использованием данных эксплуатационного контроля за состоянием оборудования.

Для достижения поставленной цели решаются следующие задачи:

- выбор объекта исследования;

- подбор эксплуатационных данных по наиболее характерным режимам работы турбоустановки;

- выбор математической модели конденсатора, обеспечивающей наиболее достоверное решение поставленной задачи, и адаптация выбранной модели к конкретным условиям;

- определение допустимого давления в конденсаторе;

- определение допустимого расхода пара в конденсатор, степени загрязнения конденсатора, ее влияния на давление в конденсатоpe и на вырабатываемую турбоустановкой мощность, а также влияния фактических присосов воздуха на давление в конденсаторе;

- проведение анализа с целью выяснения основных факторов, лимитирующих выра- ботку электрической мощности турбоустановкой;

- разработка рекомендаций по ведению режимов, обеспечивающих минимальные значения ограничений мощности.

\section{МЕТОДИКА И АЛГОРИТМЫ ИССЛЕДОВАНИЯ}

При проектировании паротурбинных установок типа ПТ считается, что конденсационный режим не является характерным, поэтому для них принимаются несколько завышенные величины удельных паровых нагрузок $\left(d_{\kappa}, \kappa \Gamma /\left(\mathrm{M}^{2} \cdot ч\right)\right.$, ) и заниженные кратности охлаждения ( $m$, т/ч воды, т/ч пара). Так, для рассматриваемой турбоустановки эти величины соответственно равны $d_{\mathrm{K}}=90,3$ и $m=30,63$. Для сравнения, у конденсаторов конденсационных турбин проектные значения этих величин находятся в пределах $d_{\mathrm{\kappa}}=30-44$ и $m=47-63$.

Таким образом, можно предположить, что в случае использования турбоустановок типа ПТ при их работе в конденсационном режиме мы имеем дело с объективно низкими проектными значениями вакуума в конденсаторе.

В конкретных условиях при проведении анализа причин ограничений мощности и разработке мероприятий по их устранению необходимо прежде всего иметь достаточно достоверные данные по параметрам, характеризующим работу конденсатора на наиболее характерных режимах эксплуатации в широком диапазоне температур и расходов охлаждающей его воды, и на основе этих данных по одной из выбранных методик определить существующие ограничения.

Фактические данные могут быть получены из данных, фиксируемых АСУ, или из суточных ведомостей контроля за состоянием работы оборудования, в частности, конденсатора, основных эжекторов и турбоустановки в целом за период не менее года. Такой подход позволяет из всего имеющегося массива данных выбрать наиболее характерные режимы эксплуатации, провести соответствующую диагностику оборудования и определить его технико-экономические показатели.

Расчетные данные могут быть получены на основе математической модели конденсатора, учитывающей его взаимодействие с эжектором. Результаты расчетов могут быть представлены в виде характеристик конденсатора для всего диапазона расходов пара в 
него. Характеристики должны быть построены на основе осредненных фактических данных для характерных режимов. В данной работе используется математическая модель конденсатора, позволяющая раздельно учитывать влияние присосов воздуха в вакуумную систему и загрязнений поверхностей теплообмена на параметры конденсации пара, а также их влияние на давление в конденсаторе [25]. Данная модель прошла верификацию на турбоустановках различных типов и используется для проведения исследований на многих ТЭЦ.

Анализ результатов производился с целью определения фактических характеристик конденсатора на основе измеренных величин и расчетных данных. В ходе проведения анализа определялась степень загрязнения поверхностей теплообмена при известных присосах воздуха в вакуумную систему. По характеристикам конденсатора, построенным для существующих режимов турбоустановки, определялись режимы, имеющие ограничения мощности.
Представленные в настоящей работе содержание и последовательность действий позволяют предложить достаточно универсальную методику определения ограничений мощности по давлению в конденсаторе паротурбинных установок.

\section{РЕЗУЛЬТАТЫ ИССЛЕДОВАНИЯ}

В качестве примера рассмотрим результаты анализа режимов работы турбоустановки ПТ-35/55-3,2 одной из ТЭЦ в России за период с июня 2019 по июль 2020 года. Анализ проводился на основе обработки записей в суточных ведомостях за указанный период и с использованием адаптированной математической модели конденсатора. Такой подход позволяет охватить всю совокупность режимов работы турбоустановки при ее эксплуатации в конкретных условиях.

Расчетные режимы турбоустановки ПТ-35/55-3,2 представлены в таблице 1.

Таблица $1^{1}$

Расчетные режимы турбоустановки ПТ-35/55-3,2 $2^{2}$

\begin{tabular}{|c|c|c|c|c|c|c|c|}
\hline \multirow{2}{*}{$\begin{array}{l}\text { Электри- } \\
\text { ческая } \\
\text { мощность/ } \\
\text { Electric } \\
\text { power }\end{array}$} & \multirow{2}{*}{$\begin{array}{c}\text { Расход пара } \\
\text { на турбину/ } \\
\text { Steam } \\
\text { consumption } \\
\text { per turbine }\end{array}$} & \multicolumn{4}{|c|}{$\begin{array}{l}\text { Регулируемые отборы/ } \\
\text { Regulated steam extractions }\end{array}$} & \multicolumn{2}{|c|}{$\begin{array}{c}\text { Параметры пара } \\
\text { в конденсаторе/ } \\
\text { Condenser steam } \\
\text { parameters }\end{array}$} \\
\hline & & \multicolumn{2}{|c|}{$\begin{array}{l}\text { производственный } \\
\text { /production } \\
\text { extraction }\end{array}$} & \multicolumn{2}{|c|}{$\begin{array}{l}\text { теплофикационный/ } \\
\text { heating extraction }\end{array}$} & $\begin{array}{c}\text { расход/ } \\
\text { consumption }\end{array}$ & $\begin{array}{l}\text { давление/ } \\
\text { pressure }\end{array}$ \\
\hline $\begin{array}{l}\text { MBT/ } \\
\text { MV }\end{array}$ & $\begin{array}{c}G_{0}, \\
\mathrm{~T} / \mathrm{\Psi} / \\
\mathrm{t} / \mathrm{h}\end{array}$ & $\begin{array}{c}G_{\text {пр }} \\
\text { т/ч/ } \\
\mathrm{t} / \mathrm{h}\end{array}$ & $\begin{array}{c}P_{\text {пр }} \\
\mathrm{MПа} / \\
\mathrm{MPa}\end{array}$ & $\begin{array}{c}G_{\text {тепл }} \\
\mathrm{T} / \mathrm{\varphi} / \\
\mathrm{t} / \mathrm{h}\end{array}$ & $\begin{array}{l}P_{\text {тепл }} \\
\mathrm{MПа/} \\
\mathrm{MPa}\end{array}$ & $\begin{array}{c}G_{\mathrm{K}} \\
\mathrm{T} / \mathrm{\varphi} / \\
\mathrm{t} / \mathrm{h}\end{array}$ & $\begin{array}{c}P_{\mathrm{K}} \\
\mathrm{M \Pi a} / \\
\mathrm{MPa}\end{array}$ \\
\hline 22,2 & 240 & 115 & 1,5 & 95 & 0,15 & 26,1 & 3,2 \\
\hline 30,6 & 240 & 130 & 1,5 & 0 & - & 101,0 & 6,4 \\
\hline 45,3 & 240 & - & 0 & 110 & 0,15 & 114,4 & 7,0 \\
\hline 39,3 & 170 & - & 0 & 0 & - & 152,2 & 9,5 \\
\hline 54,2 & 240 & - & 0 & 0 & - & 212,2 & 14,2 \\
\hline
\end{tabular}

Турбоустановка оснащена двухпоточным двухходовым конденсатором типа 35КП-2350-1 с поверхностью теплообмена 2350 м $^{2}$ из 5260 трубок из латуни Л68 наружным диаметром 23 мм и внутренним 21 мм. Номинальный расход охлаждающей воды через конденсатор $-6500 \mathrm{~m}^{3} /$ ч. В имеющейся документации нормативные характеристики конденсатора отсутствуют. Для турбоустановок данного типа предельное допустимое давление в конденсаторе, при котором допус- кается длительная их эксплуатация, принимается равным 14,2 кПа.

Для рассматриваемого случая в качестве фактических были выбраны данные, представленные в суточных ведомостях по турбоустановке за летний период эксплуатации (июль-август 2019 г.). В соответствии с целью анализа была проведена обработка данных для получения зависимостей электрической мощности, вырабатываемой турбоустановкой, от давления в конденсаторе. 
Из данных, полученных на летних режимах, были выбраны все значения давлений в конденсаторе $\left(P_{\mathrm{\kappa}}, \kappa П а\right)$, расходов пара $\left(G_{\mathrm{\kappa}}\right.$, т/ч) в него и электрической мощности, вырабатываемой турбоустановкой $\left(N_{3}, \mathrm{MBT}\right)$, соответствующие расходам охлаждающей воды в диапазоне от 7000 до 8000 т/ч (средний расход $W_{\mathrm{cp}}=7422$ т/ч) при соответствующих температурах воды на входе в конденсатор и измеренных присосах воздуха в вакуумную систему.

Для выяснения взаимозависимости давления в конденсаторе и электрической мощности, вырабатываемой турбоустановкой на летних режимах, в соответствии с данными измерений построена зависимость $N_{3}=f\left(P_{\mathrm{K}}\right)$, представленная на рис. 1.

Полученная зависимость не носит универсального характера, но позволяет установить, что в условиях эксплуатации увеличение давления в конденсаторе свыше 14,2 кПа не позволяет получить прироста мощности с увеличением расхода пара в конденсатор, при этом ограничения мощности составляют порядка 5 МВт. Наличие такого предельного давления подтверждается данными, представленными в таблице 1.

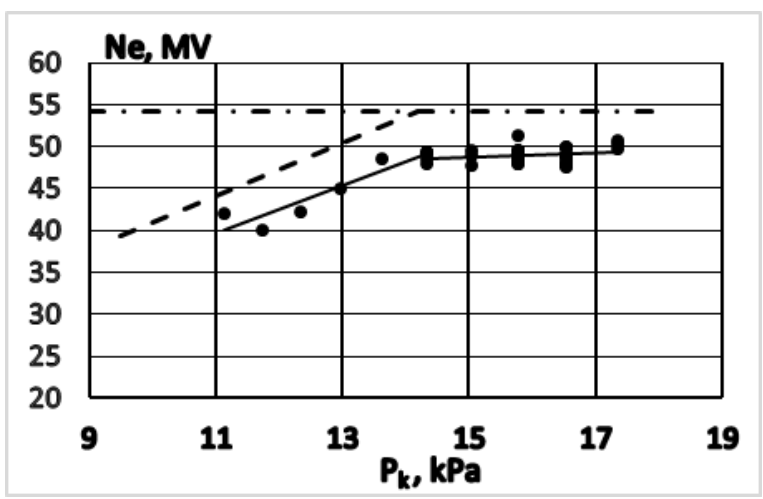

точки - даннье измерений;

сплошная линия - тренд;

штриховая линия - расчетная зависимость электрической мошности от давления в конденсаторе при температуре охлаждающей воды $t_{16}=20^{\circ} \mathrm{C}$;

штрих-пунктир - расчетная мощуность при номинальном расходе пара на турбину

Рис. 1. Зависимость электрической мощности турбоустановки ПТ-35/55-3,2 от давления в конденсаторе 35КП-2350-1 при параметрах: $W=7000-8000$ т $/ \mathbf{4} ; t_{1 \mathrm{~B} \mathrm{ср}}=26-32^{\circ} \mathrm{C}$; $G_{\mathrm{K}}=161-204 \mathrm{~T} / \mathrm{\Psi .}^{3}$

Имеющиеся данные дают основания полагать, что фактические ограничения мощности при увеличении давления в конденсаторе напрямую связаны с режимом работы основ- ного эжектора. Известно, что работа основного эжектора при температуре охлаждающего конденсата на входе в охладитель первой ступени выше $50^{\circ} \mathrm{C}$ приводит к его перегрузке и выключению, вследствие чего эжектор не в состоянии поддерживать вакуум в соответствии с паровой нагрузкой конденсатора: давление в конденсаторе растет, а мощность не увеличивается из-за уменьшения теплоперепада на турбине. На рис. 2 показана зависимость электрической мощности, вырабатываемой турбоустановкой, от температуры основного конденсата.

Представляется, что снятие указанных выше ограничений возможно при выборе режимов, обеспечивающих давление в конденсаторе ниже максимально допустимого (14,2 КПа).

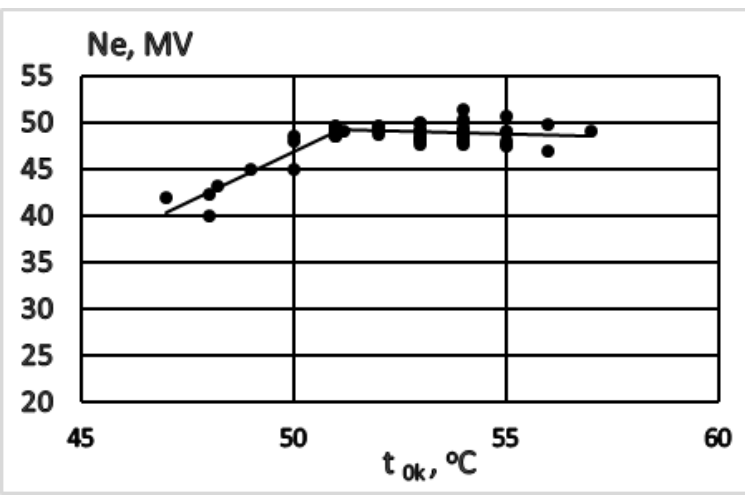

точки - данные измерений;

сплошная линия - линия тренда

Рис. 2. Зависимость электрической мощности турбоустановки ПТ-35/55-3,2 от температуры основного конденсата на входе в эжектор ЭП-3-750-2. ${ }^{4}$

На рис. 3 показано корреляционное поле $P_{\text {к }}$ от $G_{\text {к }}$ полученное на основе суточных ведомостей. На этом же рисунке представлены расчетные зависимости, построенные на основе математической модели конденсатора при средних, максимальных и минимальных температурах охлаждающей воды на входе в него и величине среднего значения расхода этой воды. При построении зависимостей $P_{\text {к }}=f\left(G_{\text {к }}\right)$ коэффициент чистоты был определен расчетным путем с использованием фактических данных.

С помощью представленных на рисунке зависимостей можно определить допустимый расход пара в конденсатор, который в данном случае равен 180 т/ч.

При заданной температуре на входе в конденсатор обеспечение приемлемого значения давления может быть осуществлено подбо- 
ром соответствующего расхода охлаждающей воды.

На рис. 4 показаны характеристики $P_{\kappa}=F(W)$ конденсатора 35КП-2350-1, построенные на основе математической модели при температуре воды на входе в $29^{\circ} \mathrm{C}$ и различных расходах пара в конденсатор. Представленные на рисунке зависимости позволяют определить условия получения номинальной мощности турбоустановки (при $G_{\text {к }}=212,2$ т/ч). В данном случае эта выработка возможна при расходе охлаждающей воды более 7400 т/ч и при степени чистоты поверхностей теплообмена, близкой к нормативной. При расчётном расходе охлаждающей воды 6500 т/ч и фактическом загрязнении расход пара в конденсатор ограничен величиной расхода в него $155 \mathrm{~T} / \mathrm{\varphi}$.

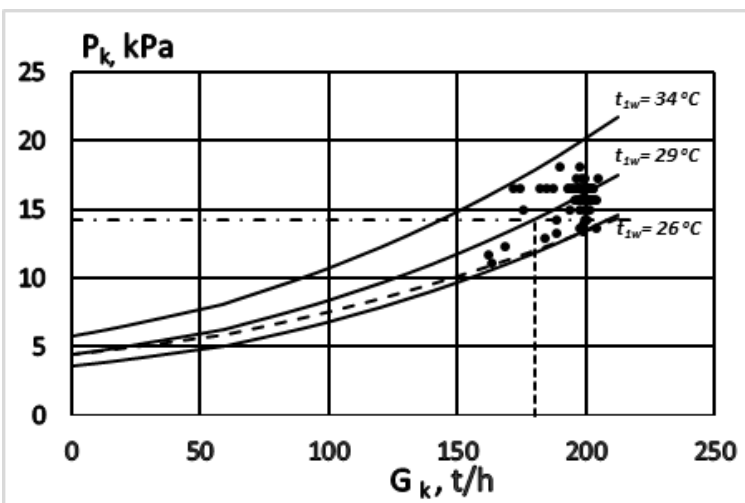

Условия построения расчетных характеристик:

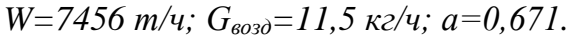

точки - данные измерений при $W_{c p}=7456 \mathrm{~m} / \mathrm{v}$ $(7000-8000 \mathrm{~m} / \mathrm{u}) ; \quad t_{c p}=29^{\circ} \mathrm{C} \quad\left(26-34^{\circ} \mathrm{C}\right)$; $G_{\kappa c p}=195,9 \mathrm{~m} / \mathrm{u}(161-204 \mathrm{~m} / \mathrm{u})$;

сплошные линии - расчетные характеристики при температурах циркводы на входе в конденcamop, $t_{16 ~ c ~}=29^{\circ} \mathrm{C}, t_{16}=34^{\circ} \mathrm{C}, t_{16}=26^{\circ} \mathrm{C}$;

пунктирная линия - нормативная характеристика конденсатора $t_{1 в \text { ср }}=29^{\circ} \mathrm{C}$;

штрих-пунктир - линия допустимого давления пара в конденсаторе;

вертикальный пунктир - линия допустимого расхода пара в конденсатор.

Рис. 3. Характеристики конденсатора 35КП-2350-1 турбоустановки ПТ-35/55-3,2.5

На рис. 4 показаны характеристики $P_{\kappa}=F(W)$ конденсатора 35КП-2350-1, построенные на основе математической модели при температуре воды на входе в $29^{\circ} \mathrm{C}$ и различных расходах пара в конденсатор. Представленные на рисунке зависимости позволяют определить условия получения номинальной мощности турбоустановки (при $G_{\kappa}=212,2$ т/ч). В данном случае эта выработка возможна при расходе охлаждающей воды более 7400 т/ч и

\footnotetext{
5, 6,7 Appendix 1
}

при степени чистоты поверхностей теплообмена, близкой к нормативной. При расчётном расходе охлаждающей воды 6500 т/ч и фактическом загрязнении расход пара в конденсатор ограничен величиной расхода в него

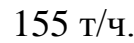

На рис. 5 показаны расчетные характеристики конденсатора 35КП-2350-1 $P_{\text {к }}=f\left(G_{\text {к }}\right)$ при заданном фактическом среднем расходе охлаждающей воды 7456 т/ч, позволяющие определить величины температур воды на входе в конденсатор, при которых возможно получение расчетной электрической мощности.

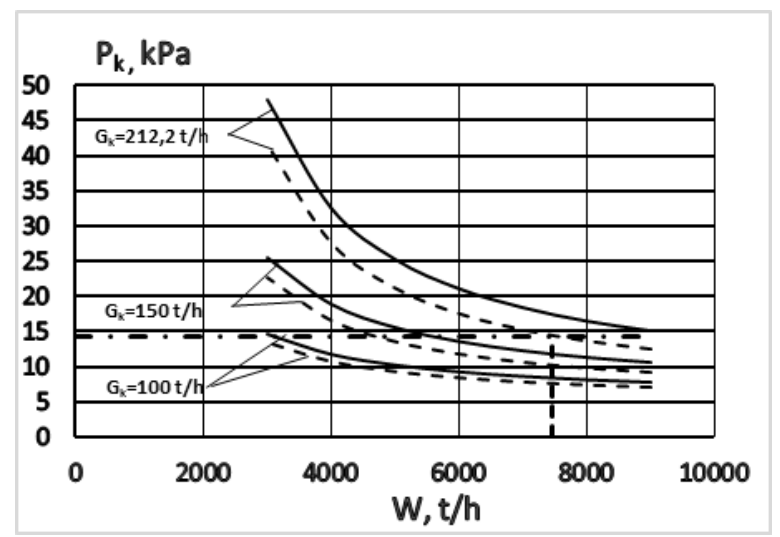

сплошные линии - расчетные характеристики при фактической степени загрязнения поверхностей теплообмена, $a=0,671$;

пунктирные линии - расчетные характеристики при нормативной степени загрязнения поверхностей теплообмена, $a=0,85$;

итрих-пунктир - линия максимального допустимого значения давления пара в конденсаторе;

вертикальный пунктир - линия наименьшего допустимого по давлению расхода охлаждающей водbl.

Рис. 4. Зависимость давления в конденсаторе 35КП-2350-1 от расхода через него охлаждающей воды при различных расходах пара в конденсатор, $t_{1 \mathrm{~B}}=29^{\circ} \mathrm{C}, G_{\text {возд }}=11,5$ кг $/$ ч. $^{6}$

На основе данных рис. 5 можно установить, что выработка номинальной мощности в рассматриваемых условиях возможна при температурах ниже $25^{\circ} \mathrm{C}$, что может быть реализовано повышением охлаждающей способности гидроохладителей.

При рассмотрении вопроса о причинах ограничений мощности весьма важным является определение величин ее недовыработки за счет наличия загрязнений на поверхностях теплообмена конденсатора.

На рис. 6 показана зависимость недовыработки мощности турбоустановкой ПТ-35/553,2 от расхода пара в конденсатор. 
Зависимость рассчитана для условий рассматриваемого режима $\left(W=7456 \mathrm{~T} / \mathrm{y}, t_{1 \mathrm{~B}}=29^{\circ} \mathrm{C}\right)$ в рабочем диапазоне расхода пара в конденсатор.

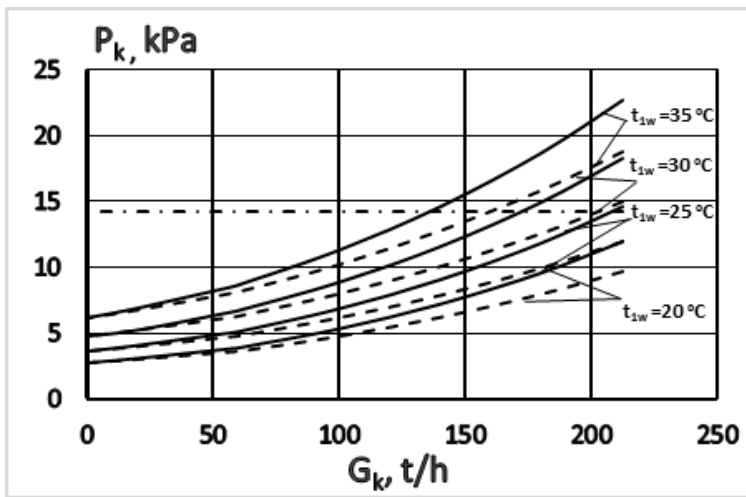

сплошные линии - расчетные характеристики при фактической степени загрязнения поверхностей теплообмена, $a=0,671$,

итриховые линии - расчетные характеристики при нормативной степени загрязнения поверхностей теплообмена, $a=0,85$;

штрих-пунктир - линия максимального допустимого значения давления пара в конденсаторе

Рис. 5. Характеристики конденсатора 35КП-2350-1, $W=7456$ т/ч, $G_{\text {возд }}=11,5$ кг $/$ ч. $^{7}$

Величины недовыработки мощности определялись по разности давлений, рассчитанных для конденсатора, имеющего фактические загрязнения $(a=0,671)$, и для технически чистого конденсатора $(a=0,85)$, с использованием нормативного графика поправок к мощности турбины на отклонение давления пара в конденсаторе.

В результате этого расчета определено, что недовыработка мощности составляет до $1,3 \%$ от номинальной электрической мощности турбоустановки.

Фактические присосы воздуха в вакуумную систему в данном случае невелики и не оказывают существенного влияния на мощность, вырабатываемую установкой.

Таким образом, выполнение исследований на основе математической модели конденсатора с использованием данных текущего контроля в определенной в данной работе последовательности позволило выяснить причины ограничений мощности турбоустановки, определить режимы, при которых возможно снятие этих ограничений, то есть в работе представлена методика, имеющая достаточно универсальный характер.

Следует отметить, что в условиях бестопливного получения острого пара на основе утилизации тепла доменного и коксового га- зов на ТЭЦ металлургических предприятий выработка максимально возможной электрической мощности всегда экономически целесообразна.

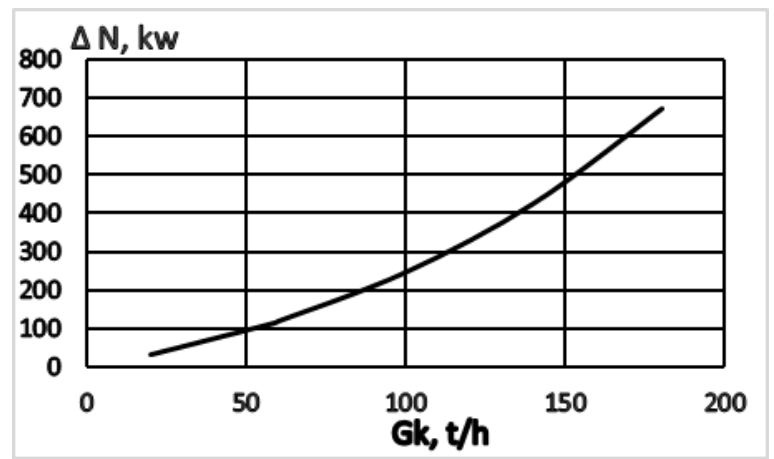

Рис. 6. Зависимость недовыработки электрической мощности турбоустановкой ПТ-35/55-3,2 от расхода пара в конденсатор, $W=7456 \mathrm{~T} / \mathrm{ч}, t_{1 \mathrm{~B}}=29^{\circ} \mathrm{C}, G_{\text {возд }}=11,5$ кг $/ \mathrm{ч}^{8}$

\section{ЗАКЛЮЧЕНИЕ}

- В результате исследования выбранного объекта на основе математической модели его конденсатора получены следующие основные результаты. Предложена и апробирована методика выяснения причин ограничений мощности турбоустановки на основе уточненной математической модели ее конденсатора с использованием данных эксплуатационного контроля за состоянием оборудования. В соответствии с предложенной методикой выполнен анализ конкретных режимов эксплуатации турбоустановки в определенной последовательности. На основе суточных ведомостей текущего контроля выбраны данные, характеризующие режимы работы конденсатора за период наибольших значений давления в конденсаторе. На основе фактических данных построены зависимости мощности, вырабатываемой турбоустановкой, от величины давления в конденсаторе. Установлена величина предельного допустимого давления в конденсаторе, которое для турбоустановки данного типа равно 14,2 кПа. Показано, что превышение указанного давления приводит к ограничению электрической мощности турбоустановки. Причиной такого ограничения является переход основного эжектора на нештатный режим работы вследствие повышенной температуры основного конденсата, поступающего в его охладитель первой ступени. На основе фактических данных с помощью математической модели конденсатора построены зависимости давления в конденсаторе от расхода в него пара. С по- 
мощью построенных зависимостей и установленной ранее величины допустимого давления определена величина максимально допустимого расхода пара в конденсатор в рассматриваемых условиях.

- Определены области режимов работы конденсатора по расходу охлаждающей конденсатор воды при заданной средней ее температуре, обеспечивающие выработку номинальной и даже большей ее величины электрической мощности.

- Определены области режимов работы конденсатора от температуры охлаждающей воды на входе в него при заданном ее расходе.

- Определены величины недовыработки мощности турбоустановкой в зависимости от расхода пара в конденсатор на режимах, соответствующим фактическим данным.

Использованные в настоящей работе содержание и последовательность действий представляют собой достаточно универсальную методику определения ограничений мощности по давлению в конденсаторе паротурбинных установок.

\section{APPENDIX 1 (ПРИЛОЖЕНИЕ 1)}

1,2 Table 1. Calculation modes of turbine unit PT35/55-3,2.

${ }^{3}$ Fig. 1. Electric power dependence of PT-35/55-3,2 turbo plant on condenser pressure, $\mathrm{W}=7000-8000 \mathrm{t} / \mathrm{h}$; $t_{1 \mathrm{~B} \mathrm{cp}}=26-32^{\circ} \mathrm{C} ; G_{\mathrm{K}}=161-204 \mathrm{t} / \mathrm{h}$. (the points - measured data; solid line - trend; the dashed line - estimated power capacity dependence on condenser pressure at cooling water temperature $\mathrm{t} 1 \mathrm{c}=20^{\circ} \mathrm{C}$; the dashdotted line - estimated power at nominal steam consumption per turbine).

${ }^{4}$ Fig. 2. Dependence of electric power of turbine unit PT-35/55-3.2 on the temperature of primary condensate at the inlet of the ejector EP-3-7502 type (the points - measured data; the solid line - trend)

${ }^{5}$ Fig. 3. Characteristics of condenser 35KP-23501 of turbine unit PT-35/55-3,2. (the conditions of design characteristics $\mathrm{W}=7456 \mathrm{t} / \mathrm{h} ; \mathrm{G}_{\text {возд }}=11,5 \mathrm{~kg} / \mathrm{h}$; $\mathrm{a}=0,671$; - measurement data $\mathrm{W}_{\mathrm{cp}}=7456 \mathrm{t} / \mathrm{h}(7000$ $8000 \mathrm{t} / \mathrm{h}) ; \mathrm{t}_{\mathrm{cp}}=29^{\circ} \mathrm{C}\left(26-34^{\circ} \mathrm{C}\right) ; \mathrm{G}_{\mathrm{\kappa} \mathrm{cp}}=195,9 \mathrm{t} / \mathrm{h}(161-$ $204 \mathrm{t} / \mathrm{h}$ ); the solid lines - design characteristics at temperatures of circulating water at condenser inlet $\mathrm{t}_{1 \mathrm{~B} \mathrm{cp}}=29^{\circ} \mathrm{C}, \mathrm{t}_{1 \mathrm{~B}}=34^{\circ} \mathrm{C}, \mathrm{t}_{1 \mathrm{~B}}=26^{\circ} \mathrm{C}$; the dashed line normative condenser characteristic at $\mathrm{t}_{\mathrm{B} \mathrm{B}_{\mathrm{cp}}}=29^{\circ} \mathrm{C}$; the dash-dotted line - line of permissible pressure in the condenser; the vertical dotted line - line of allowable steam flow in condenser).

${ }^{6}$ Fig. 4. Dependence of pressure in the 35KP-2350-1 condenser on the flow rate of cooling water through it at different flow rates of steam into the condenser, $t_{1 \mathrm{~B}}=29^{\circ} \mathrm{C}, G_{\text {возд }}=11,5 \mathrm{~kg} / \mathrm{h}$. (the solid lines - calculated characteristics at actual degree of pollution of heat exchange surfaces, $\mathrm{a}=0.671$; the dashed lines calculated characteristics at normative degree of pollution of heat exchange surfaces, $a=0.85$; the dashdotted line - line of maximum permissible value of steam pressure in condenser; the vertical dotted line line of the least permissible by pressure cooling water discharge).

${ }^{7}$ Fig. 5. Characteristics of condenser 35KP-23501, $W=7456 \mathrm{t} / \mathrm{h}, G_{\text {возд }}=11.5 \mathrm{~kg} / \mathrm{h}$. (the solid lines calculated characteristics at actual degree of pollution of heat exchange surfaces, $a=0,671$; the dashed lines calculated characteristics at normative degree of pollution of heat exchange surfaces, $a=0,85$; the dashdotted line - line of maximum allowable value of steam pressure in condenser).

${ }^{8}$ Fig. 6. Dependence of power underproduction by a PT-35/55-3,2 turbine unit on steam consumption in the condenser, $W=7456 \mathrm{t} / \mathrm{h}, \quad t_{1 \mathrm{~B}}=29^{\circ} \mathrm{C}, \quad G_{\text {возд }}=11,5$ $\mathrm{kg} / \mathrm{h}$.

\section{Литература (references)}

[1] Chuang C.C., Sue D.C. Performance effects of combined cycle power plant with variable condenser pressure and loading. Energy, 2005, vol. 30, no. 10, pp. 1793-1801. doi: 10.1016/j.energy.2004.10.003

[2] Geete A., Khandwawala A.I. Thermodynamic analysis of $120 \mathrm{MW}$ thermal power plant with combined effect of constant inlet pressure (124.61 bar) and different inlet temperatures. Case Studies in Thermal Engineering, 2013, vol. 1, no. 1, pp. 17-25. doi: 10.1016/j.csite.2013.08.001

[3] Mihajskij D.V. Issledovanie vliyaniya rezhimov raboty NPK na effektivnost' raboty energoblokov [Investigation of the influence of operating modes of a low-potential complex on the power units efficiency]. Vostochno-evropejskij zhurnal peredovyh tekhnologij - Eastern European Journal of Advanced Technologies, 2005, no. 3, pp. 122-125. (In Russian)

[4] Aronson K.E., Murmanskii I.B., Brodov Y.M., Stepanov M.Y. Steam pressure's influence in the condenser on the efficiency indicators of the $t$ 100-12.8 turbine when operating with a fully closed diaphragm according to the thermal diagram. Thermal Engineering, 2020, vol. 67, no. 12, pp. 892-895. doi: $10.1134 / \mathrm{S} 0040601520120022$

[5] RD 153-34.1-22.508-2001. Metodicheskie ukazaniya po opredeleniyu obespechennosti elektrostancij cirkulyacionnymi sistemami vodosnabzheniya [Russian Guideline 153-34.122.508-2001. Methodological guidelines for determining the provision of power plants with circulating water supply systems]. Moscow, SPO ORGRES, 2001.

[6] Zorner W., Drosdiok A. Functional and Thermodynamic Control of Steam Turbine Conden- 
sers and their Peripheral Systems // ASME/IEEE Power Generation Conference. USA. 1988.

[7] Kalatuzov V.A. Nizkopotencial'naya chast' teplovyh elektrostancij odna iz prichin ogranicheniya ih moshchnosti [The low-potential part of thermal power plants is one of the reasons for the limitation of their capacity]. Energosberezhenie $i$ vodopodgotovka - Energy saving and water treatment, 2010, no. 3(65), pp. 34-37. (In Russian)

[8] $\mathrm{Hu}$ N.-S., He N.-N., Hu S. Fault diagnosis of the steam turbine condenser system based on SOM neural network. Proc. Int. Conf. on Machine Learning and Cybernetics, 2003, pp. 12221225. doi: 10.1109/ICMLC.2003.1259673.

[9] Laskowski R., Smyk A., Lewandowski J., Rusowicz A. Cooperation of a Steam Condenser with a Low-pressure Part of a Steam Turbine in Off-design Conditions. American Journal of Energy Research, 2015, vol. 3, no. 1, pp. 13-18.

[10] Standards for steam surface condensers. 11Th edition, USA: Heat Exchange Institute, 2012. $108 \mathrm{p}$.

[11] Nadig R. Evacuation systems for steam surface condensers: vacuum pumps or steam jet air ejectors. [Proc. ASME 2016 Power Conf. POWER 2016, Charlotte, NC, June 26-30], 2016. doi: 10.1115/POWER2016-59067

[12] ESDU Ejectors and Jet Pumps Data Item 86030, 1986. ESDU International Ltd, London, UK.

[13] Croll W. S. Keeping steam ejectors. Chem. Eng, 1998, vol. 105, no. 4, pp. 108-112.

[14] Brodov Y.M., Aronson K.E., Ryabchikov A.Y., Nirenshteyn M.A. Current state and trends in the design and operation of water-cooled condensers of steam turbines for thermal and nuclear power stations. Thermal Engineering, 2019, vol. 66, no. 1, pp. 16-26. doi: 10.1134/s0040601519010026.

[15] Zorner W., Andreae K.-H. Diagnosesystem zur Betriebsuberwachung von Dampfturbinenanlagen. $V G B$. Kraftwerkstechnik, 1991, p.6.

[16] Michelis C., Rinaldi C., Sampietri C., Vario R. Condition monitoring and assessment of power plant components. Power Plant Life Management and Performance Improvement, 2011, pp. 38-10. doi: 10.1533/9780857093806.1.38

[17] Nagel C. Steam Turbine Condition Monitoring (TCM) [Proc. of the ASME Power Conference - San Antonio, TX; United States; 17 July 2007 through 19 July 2007], 2007, pp. 327-334. doi: 10.1115/POWER2007-22062
[18] Rusinowski H. Mathematical model of a steam turbine for the thermal diagnostics system [Proc. in the 2016 17th International Carpathian Control Conference (ICCC)], 2016, pp. 630-634. doi: 10.1109/CarpathianCC.2016.7501172

[19] Salamati S.A., Salamati S.M., Salmasi F.R. Experimental identification and verification for a comprehensive model of multi-shaft combined cycle power plant [Proc. in the 2018 IEEE/IAS 54th Industrial and Commercial Power Systems Technical Conference (I\&CPS)], 2018, pp. 1-8. doi: 10.1109/ICPS.2018.8467714

[20] Huzarek M., Duzinkiewicz K., Piotrowski R. Static model of steam/water cycle for thermal and economic analysis [Proc. in the 2015 20th International Conference on Methods and Models in Automation and Robotics (MMAR)], 2015, pp. 65-86. doi: 10.1109/MMAR.2015.7283885

[21] Salamati S.A., Salamati S.M., Salmasi F.R. Experimental identification and verification for a comprehensive model of multi-shaft combined cycle power plant [Proc. in 2018 IEEE/IAS 54th Industrial and Commercial Power Systems Technical Conference (I\&CPS)], 2018, pp. 1-8. doi: 10.1109/ICPS.2018.8467714

[22] Laskowski R.M. A mathematical model of a steam condenser in off-design operation. Journal of Power Technologies, 2012, no. 92, pp. 101-108.

[23] Géczy G. Dynamic simulation of heater condenser and extraction condenser steam turbine. Energy [Proc. in Energy (IYCE), 2013 4th International Youth Conference], 2013, pp. 1-5. doi: 10.1109/IYCE.2013.6604195

[24] Aronson K.E., Murmansky B.E., Novoselov V.B., Brodov YU.M., Sosnovsky A.YU., Murmanskii I.B., Izotin D.A. Adaptation of algorithms for diagnostics of steam turbine unit equipment to specific conditions at thermal power stations. Thermal Engineering, 2020, vol. 67, no. 11, pp. 800-804. doi: 10.1134/S0040601520110014

[25] Shempelev A.G., Sushchih V.M, Iglin P.V. O rezul'tatah sopostavleniya raschetnyh i normativnyh harakteristik kondensatorov paroturbinnyh ustanovok v shirokom diapazone ih parovyh nagruzok [On the results of comparing the design and standard characteristics of steam turbine condensers in a wide range of their steam loads]. Energetik - Power Engineer, 2015, no. 10, pp. 60-64.

\section{Сведения об авторе}

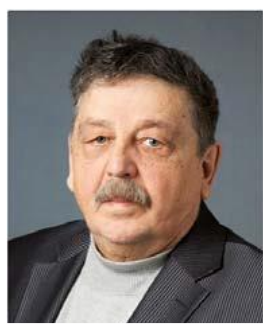

Шемпелев Александр Георгиевич, д.т.н., доцент, профессор кафедры теплотехники и гидравлики ВятГУ. Область научных интересов: теплообменные аппараты, повышение эффективности работы ТЭЦ. E-mail: agshem@mail.ru 